\title{
Analytical Solution of Richards' Equation for Application in Automatic Irrigation Systems
}

\author{
Ise Ise Ekpoudom, Anamekere Ime Jacob, Umana Thompson Itaketo
}

Department of Electrical, Electronic and Computer Engineering, University of Uyo, Uyo, Nigeria

Email address:

highpointer7@yahoo.com (I. I. Ekpoudom), jacobsanams@yahoo.com (A. I. Jacob), engr1easy@yahoo.com (U. T. Itaketo)

\section{To cite this article:}

Ise Ise Ekpoudom, Anamekere Ime Jacob, Umana Thompson Itaketo. Analytical Solution of Richards' Equation for Application in Automatic Irrigation Systems. International Journal of Theoretical and Applied Mathematics. Vol. 3, No. 5, 2017, pp. 163-166. doi: $10.11648 /$ j.ijtam.20170305.12

Received: February 20, 2017; Accepted: March 14, 2017; Published: October 28, 2017

\begin{abstract}
This paper presents the analytical solution to Richards' equation of hydrology for unsaturated soils. In order to facilitate the design and analysis of a real-time automatic irrigation system, an accurate model must be developed for the system. Richard's equation of water hydrology may be used to model part of the irrigation system. The major problem with the application of Richard's equation is in the linearization of the non-linear partial differential equation (PDE). In this paper, an empirical relationship stated by Gardner [1] was used to linearize the nonlinear PDE. The solution for the PDE was obtained using separation of variables technique.
\end{abstract}

Keywords: Richards’ Equation, Partial Differential Equations, Irrigation, Hydrology, Soil Moisture

\section{Introduction}

In order to develop an analytical model for automatic irrigation systems, it is necessary to obtain the analytical solution to Richards' equation of water hydrology for unsaturated soils. Water flow is governed by Richards' equations while constitutive laws are typically supplied by the van Genucthen model [2] Over the years, a lot of works have been done in the area of manufacturing and installation of automatic irrigation system but none has been seen in the area of development of a complete analytical model for a real-time automatic irrigation system. Consequently, to adequately design a real-time automatic irrigation system, an accurate analytical model is necessary to facilitate the simulation, prediction and control analysis of the system which largely depends on the development of accurate soil moisture content and plant water uptake models. Richard's equation of water hydrology, which can be used to model part of the irrigation system, is an important equation which governs the flow of water into the soil. In other words, modelling one-dimensional flow process in unsaturated soils is usually based on the solutions of Richards' equation [3].

\section{Automated Irrigation System}

The main purpose of irrigation system should not only be to supply irrigation water to plants, but to do so in a very efficient and adequate way. Bearing in mind that excess or inadequate supply of irrigation water to plant causes damage in one way or the other to plant, there is need for the use of an automatic irrigation system for effective and adequate supply of irrigation water to plants [4].

An automatic irrigation system is an irrigation system designed to be operated by a programmable controller which is programmed to operate automatically with minimum manual intervention, especially in the sensing of the level of soil moisture content. It consists of the following components: piping, sprinkler heads or dripper heads and other parts that are associated with a standard irrigation system along with automated components such as controller, soil moisture sensor and supervisory control system. An automatic soil moisture based irrigation system uses the soilwater sensor to sense the level of soil moisture present in the soil, so that when the soil becomes dry to certain level, say the critical moisture content $(\theta c)$, the irrigation system operate and provide the soil with water. The sensor also acts as rain sensor which does not turn on the irrigation system 
when the soil moisture level is enough for plant survival [5].

Over the years, a lot of works have been done in the area of manufacturing and installation of automatic irrigation system but none has been seen in the area of development of a complete analytical model for a real-time automatic irrigation system. Consequently, to adequately design a realtime automatic irrigation system, an accurate analytical model is necessary. Richard's equation of water hydrology is an important equation which governs the flow of water into the soil. The major problem usually encountered with the application of Richard's equation in the analysis of the flow of water into the soil is in the linearization of the non-linear partial differential equation. Hence, it is necessary to develop an analytical solution for Richards' equation [3].

\section{Derivation of Richards' Equation}

Considering an elemental volume of the soil having the edges of length $\Delta x, \Delta y$ and $\Delta z$. The difference between the volume of water flowing into the elemental volume and volume of water that is flowing out is equal to the water content in the element within time change of $\Delta t$. The rate of inflow in $x$ direction is $q_{x}$. Assuming that the rate of change for $q_{x}$ is continuous so the rate of outflow is

$$
q_{x}+\frac{\partial q_{x}}{\partial x} \Delta x
$$

The inflow volume is

$$
q_{x} \Delta y \Delta z \Delta t
$$

And the outflow volume is:

$$
\left(q_{x}+\frac{\partial q_{x}}{\partial x} \Delta x\right) \Delta y \Delta z \Delta t
$$

The difference between inflow and outflow in $x$ direction is:

$$
-\frac{\partial q_{x}}{\partial x} \Delta x \Delta y \Delta z \Delta t
$$

Similarly, the differences between inflow and outflow in $y$ and $\mathrm{z}$ direction are:

$$
\begin{aligned}
& -\frac{\partial q_{y}}{\partial y} \Delta x \Delta y \Delta z \Delta t \\
& -\frac{\partial q_{z}}{\partial z} \Delta x \Delta y \Delta z \Delta t
\end{aligned}
$$

The change in water content for the entire representative soil volume is the sum of Equations 4, 4 and 6, meaning that:

$$
\frac{\Delta \theta}{\Delta t} \Delta x \Delta y \Delta z \Delta t=-\left(\frac{\partial q_{x}}{\partial x}+\frac{\partial q_{y}}{\partial y}+\frac{\partial q_{z}}{\partial z}\right) \Delta x \Delta y \Delta z \Delta t
$$

As $t \rightarrow 0$, Equation 7 becomes

$$
\frac{\partial \theta}{\partial t}=-\left(\frac{\partial q_{x}}{\partial x}+\frac{\partial q_{y}}{\partial y}+\frac{\partial q_{z}}{\partial z}\right)
$$

Also, from the derivation of Darcy's equation for saturated soils, the following relationship holds [6]:

$$
\nabla q=-K \frac{\partial H}{\partial z}
$$

Where $\mathrm{H}$ represents the total hydraulic head, $\mathrm{z}$ represents the vertical distance from the soil surface downwards and $K$ is the soil hydraulic conductivity.

Combining Equation 8 with Equation 9, Equation 10 is obtained as:

$$
\frac{\partial \theta}{\partial t}=-\left(\frac{\partial}{\partial x}\left(K(h) \frac{\partial H}{\partial x}\right)+\frac{\partial}{\partial y}\left(K(h) \frac{\partial H}{\partial y}\right)+\frac{\partial}{\partial z}\left(K(h) \frac{\partial H}{\partial z}\right)\right)
$$

Considering one dimensional analysis and assuming that the soil is isotropic then

$$
\frac{\partial \theta}{\partial t}=\frac{\partial}{\partial z}\left(K(h) \frac{\partial h}{\partial z}\right)-\frac{\partial K}{\partial z}
$$

In non-rigid soil when the soil swells or shrinks due to the water content, Equations 11 stated cannot be used. Considering water diffusion through the elemental soil volume, a parameter called soil water diffusivity can be introduced into the above equation [7]. Let this parameter be defined as:

$$
\frac{\partial h}{\partial z}=\frac{\partial h}{\partial \theta} \frac{\partial \theta}{\partial z}
$$

Substituting Equation 12 in Equation 11, Equation 13 is obtained

$$
\frac{\partial \theta}{\partial t}=\frac{\partial}{\partial z}\left(K(h) \frac{\partial h}{\partial \theta} \frac{\partial \theta}{\partial z}\right)-\frac{\partial K}{\partial z}
$$

The connection between soil water diffusivity $(D)$, soil moisture content $(\theta)$, and hydraulic conductivity $(K)$ is given as:

$$
K(\theta)=D(\theta) \frac{\partial \theta}{\partial h}
$$

Substituting Equation 14 in Equation 13, Equation 15 is obtained

$$
\frac{\partial \theta}{\partial t}=\frac{\partial}{\partial z}\left(D(\theta) \frac{\partial \theta}{\partial z}\right)-\frac{\partial K}{\partial z}
$$

Equation 15 called Richard's equation of water hydrology for unsaturated soils [8]

\section{Methodology}

\section{Analytical Solution of Richard's Equation}

The main objective of this section is to obtain an analytical solution for the boundary valued problem shown in Equations 15, that is, the equation is subject to appropriate boundary and initial conditions. The soil moisture, $\theta$, is a function of both time and location [9]; therefore, the governing equation is partial differential equation (PDE). The governing equation cannot be integrated directly, since it is a PDE and not an ordinary differential equation (ODE). One way to solve this kind of boundary valued problem is to use a technique called "separation of variables". Another way is to 
transform the PDE into a simpler equation, by carrying out operations on the PDE that will convert it into an ODE or an algebraic expression that can be easily solved [10]. These operations are called integral transforms; they transform one or more of the derivatives into an algebraic function.

In summary, the following steps will be taken to solve the problem analytically.

i. Provide a function of hydraulic conductivity and soil moisture content (Richard's equation).

ii. Establish initial and boundary conditions.

iii. Perform a change of variables to linearize Richards' equation.

iv. Solve this new PDE for the steady-state solution.

v. Use separation of variables.

vi. Transform back to the original variables.

Hydraulic Conductivity and Soil Moisture Content

Gardner's equation [1] shown in Equation 16 will be used to linearize the nonlinear PDE shown in Equation 15.

$$
S_{e}=\frac{\theta-\theta_{d}}{\theta_{s}-\theta_{d}}=K_{r}
$$

Where $S_{e}=K_{r}$ is the relative hydraulic conductivity. The relative hydraulic conductivity is expressed as shown in Equation 17.

$$
K_{r}=\frac{K}{K_{S}}[11]
$$

Where $K$ is the true hydraulic conductivity of the soil and $K_{S}$ is the hydraulic conductivity when the soil becomes saturated with water.

\section{Initial and Boundary Conditions}

Suppose Equation 15 is subjected to the following initial and boundary conditions

i. $\theta(z, 0)=0(z=0, t=0)$ Initial Condition

ii. $\theta(z, 0)=\theta_{d}(z=0, t>0)$ Lower Boundary Condition (Phreatic surface)

iii. $\theta(L, t)=\theta_{s}-\theta_{d}(z=L, t>0)$ Upper Boundary Condition (root zone),

Where $L$ is plant root zone from the phreatic surface, $\theta_{d}$ is moisture content when the soil is dry, and $\theta_{s}$ is the saturated moisture content.

\section{Linearization of Richard's Equation}

Equation 15 is a nonlinear PDE and as such, it is necessary to simplify the equation by applying change of variables to the $\frac{\partial K}{\partial z}$ term [12].

Combining Equations 16 and 17, the true hydraulic conductivity of the soil can be expressed as:

$$
K=\frac{K_{s}}{\theta_{s}-\theta_{d}} \theta-\frac{K_{s} \theta_{d}}{\theta_{s}-\theta_{d}}
$$

Differentiating both sides of Equation 18 with respect to $\mathrm{z}$,

$$
\frac{\partial K}{\partial z}=\frac{K_{S}}{\theta_{S}-\theta_{d}} \frac{d \theta}{d z}
$$

If a constant $\mathrm{c}$ is defined as follows:

$$
\mathrm{c}=\frac{K_{S}}{\theta_{s}-\theta_{d}}
$$

Then Equation 20 becomes

$$
\frac{\partial K}{\partial z}=c \frac{d \theta}{d z}
$$

Substituting Equation 21 in Equation 15, the nonlinear PDE is linearized as:

$$
\frac{\partial \theta}{\partial t}=D \frac{\partial^{2} \theta}{\partial z^{2}}+c \frac{\partial \theta}{\partial z}
$$

Application of Separation of Variables to Richard's Equation

In order to solve the linearized Equation 22, the method of separation of variables is applied and the following steps are adopted.

Step 1: Assume that $\theta(z, t)=Z(z) T(t)$, i.e., $\theta$ can be written as the product of two functions, one depends only on $z$, the other depends only on $t$. This leads to

$$
\begin{aligned}
& D T \frac{d^{2} Z}{d z^{2}}+c \frac{d Z}{d z}=Z \frac{d T}{d t} \\
& \frac{1}{Z}\left(D \frac{d^{2} Z}{d z^{2}}+c \frac{d Z}{d z}\right)=\frac{1}{T} \frac{d T}{d t}
\end{aligned}
$$

The only nontrivial solution occurs when the left- and right-hand sides of Equation 23 are set to the same arbitrary constant, $\lambda[13]$. Thus,

$$
\begin{gathered}
\frac{1}{Z}\left(D \frac{d^{2} Z}{d z^{2}}+c \frac{d Z}{d z}\right)=\frac{1}{T} \frac{d T}{d t}=\lambda \\
D \frac{d^{2} Z}{d z^{2}}+c \frac{d Z}{d z}-\lambda Z=0, \frac{d T}{d t}-\lambda \mathrm{T}=0
\end{gathered}
$$

This leads to the following characteristics equations

$$
D r^{2}+c r-\lambda=0, q-\lambda=0
$$

The solutions for Equation 25 are

$$
r_{1}=\frac{-c+\sqrt{c^{2}+4 D \lambda}}{2 D}, r_{2}=\frac{-c-\sqrt{c^{2}+4 D \lambda}}{2 D}, q=\lambda
$$

The general solution to $\theta(z, t)$, become

$$
\begin{gathered}
Z=A_{1} e^{r_{1} z}+A_{2} e^{r_{2} z}, T=e^{q t} \\
\theta(z, t)=Z T=\left(A_{1} e^{r_{1} z}+A_{2} e^{r_{2} z}\right) e^{q t}
\end{gathered}
$$

Where $A_{1}$ and $A_{2}$ are determined by initial and boundary conditions.

For a physically realizable system, $<0$. To keep the problem real and eliminate any complex part in the solution, then the choice,

$$
\lambda=\frac{-c^{2}}{4 D}+\left(\frac{\pi}{L}\right)^{2}
$$

Substituting Equation 28 in Equation 26,

$$
r_{1}=\frac{-c}{2 D}+\frac{\pi}{2 D L}, r_{2}=\frac{-c}{2 D}-\frac{\pi}{2 D L}, q=\frac{-c^{2}}{4 D}+\left(\frac{\pi}{L}\right)^{2}
$$

Also, substituting Equation 29 into Equation 27, the exact solution is expressed as: 


$$
\theta(z, t)=\left(A_{1} e^{\frac{\pi z}{2 D L}}+A_{2} e^{-\frac{\pi z}{2 D L}}\right) e^{\frac{-c z}{2 D}-\frac{c^{2} t}{4 D}+\frac{\pi^{2} t}{L^{2}}}
$$

Applying the initial and boundary conditions stated Section 3. 1. 3 to Equation 30, the coefficients of the solution is thus determined as:

$$
\begin{gathered}
A_{1}=\frac{\theta_{d}}{e^{-\frac{c^{2} t}{4 D}+\frac{\pi^{2} t}{L^{2}}}}-\frac{\left[\left(\theta_{S}-\theta_{d}\right)-\theta_{d} e^{\left.-\frac{c L}{2 D}+\frac{\pi}{2 D}\right]}\right.}{\left(e^{-\frac{c L}{2 D}-\frac{c^{2} t}{4 D}+\frac{\pi^{2} t}{L^{2}}-\frac{\pi}{2 D}}-e^{-\frac{c L}{2 D}+\frac{\pi^{2} t}{L^{2}}+\frac{\pi}{2 D}}\right)} \\
A_{2}=\frac{\left[\left(\theta_{S}-\theta_{d}\right)-\theta_{d} e^{-\frac{c L}{2 D}+\frac{\pi}{2 D}}\right]}{\left(e^{-\frac{c L}{2 D}-\frac{c^{2} t}{4 D}+\frac{\pi^{2} t}{L^{2}}-\frac{\pi}{2 D}}-e^{-\frac{c L}{2 D}+\frac{\pi^{2} t}{L^{2}}+\frac{\pi}{2 D}}\right)}
\end{gathered}
$$

Thus, the final expression for the exact solution is given as:

$$
\begin{aligned}
& \theta(z, t)=\left[\frac{\theta_{d} e^{\frac{\pi z}{2 D L}}}{e^{-\frac{c^{2} t}{4 D}+\frac{\pi^{2} t}{L^{2}}}}-\frac{\left[\left(\theta_{S}-\theta_{d}\right) e^{\frac{\pi z}{2 D L}}-\theta_{d} e^{-\frac{c L}{2 D}+\frac{\pi}{2 D}+\frac{\pi z}{2 D L}}\right]}{\left(e^{-\frac{c L}{2 D}-\frac{c^{2} t}{4 D}+\frac{\pi^{2} t}{L^{2}}-\frac{\pi}{2 D}}-e^{-\frac{c L}{2 D}+\frac{\pi^{2} t}{L^{2}}+\frac{\pi}{2 D}}\right)}+\right. \\
& \left.\frac{\left[\left(\theta_{S}-\theta_{d}\right) e^{-\frac{\pi z}{2 D L}}-\theta_{d} e^{-\frac{c L}{2 D}+\frac{\pi}{2 D}-\frac{\pi z}{2 D L}}\right]}{\left(e^{-\frac{c L}{2 D}-\frac{c^{2} t}{4 D}+\frac{\pi^{2} t}{L^{2}}-\frac{\pi}{2 D}}-e^{-\frac{c L}{2 D}+\frac{\pi^{2} t}{L^{2}}+\frac{\pi}{2 D}}\right)}\right] e^{-\frac{c z}{2 D}-\frac{c^{2} t}{4 D}+\frac{\pi^{2} t}{L^{2}}}
\end{aligned}
$$

\section{Conclusion}

The exact solution of Richards' equation as obtained through linearization of the PDE and separation of variables method has been presented in this paper. The expression for the solution can be used to develop the model for Soil Moisture Content. Such a model is necessary for the design of a real-time automatic irrigation system.

\section{References}

[1] Gardner, W. "Some Steady-State Solutions of the Unsaturated Moisture Low Equation with Application to Evaporation from a Water Table", Soil Science, Vol. 85, 1958, pp. 228-232.
[2] Kuraz, M., Mayer, P. and Pechm P. "Solving the Non-linear Richards' Equation Model with Adaptive Domain Decomposition", Journal of Computational and Applied Mathematics, Vol. 270, 2014, pp 2-11.

[3] Bouchemella, S., Seridi, A. and Alimi-Ichola, I. "Numerical Solution of Water Flow in Unsaturated Soils: Comparative Study of Different Forms of Richards' Equations" European Journal of Environmental and Civil Engineering, Vol. 9, 2014, pp 1.

[4] Bhosale P. A. and Dixit V. V. "Water Saving-irrigation Automatic Agricultural Controller", International Journal of Science and Technology, Volume 1, Number 2, 2012, pp. 3436 .

[5] Israelsen O. W. and Hansen V. E., Irrigation Principle and Practice, John Wiley and Son Inc., 3rd Edition, New Delhi, $\mathrm{p}$ 171, 2011.

[6] Hillel, D., Introduction to Environmental Soil Physics, Academic Press, Hardcover, Boston, 2003.

[7] Philip, J. R., "Hydrostatics and Hydrodynamics in Selling Soils", Water Resources, Res. 5, 1969, pp 1070-1077.

[8] Celia, M. A., Bouloutas, E. T. and Zarba, R. L. "A General Mass-Conservative Numerical Solution for the Unsaturated Flow Equation", Water Resources Research, Volume 26, Number 7, 1990, pp. 1483-1696.

[9] Majumdar D. K., Irrigation Water Management: Principles and Practice, Eastern Economy Edition, Prentice - Hall of India Private Limited. New Delhi, 2006.

[10] Tracy, F. "Clean Two- And Three-Dimensional Analytical Solutions of Richards' Equation for Testing Numerical Solvers", Water Resources Research, Vol. 42, Number 8, 2006, pp. 3106-109.

[11] Tracy, F. "Three-Dimensional Analytical Solutions of Richards' Equation for a Box-Shaped Soil Sample with Piecewise-Constant Head Boundary Conditions on the Top", Journal of Hydrology, Vol. 336, 2007, pp. 391-400.

[12] Warrick, A., Soil Water Dynamics, Oxford University Press, Paperback, New York, 2003.

[13] Genuchten, M. "A Closed-Form Equation for Producing the Hydraulic Conductivity of Unsaturated Soils", Soil Science American Journal, Vol. 44, 1980, pp. 892-898. 